\title{
BOUNDARY GROWTH OF GENERALIZED RIESZ POTENTIALS ON THE UNIT BALL IN THE VARIABLE SETTINGS
}

\author{
Yoshihiro Mizuta, Takao Ohno and Tetsu Shimomura \\ 4-13-11 Hachi-Hon-Matsu-Minami, Higashi-Hiroshima 739-0144, Japan; \\ yomizuta@hiroshima-u.ac.jp \\ Oita University, Faculty of Education \\ Dannoharu Oita-city 870-1192, Japan; t-ohno@oita-u.ac.jp \\ Hiroshima University, Graduate School of Education, Department of Mathematics \\ Higashi-Hiroshima 739-8524, Japan; tshimo@hiroshima-u.ac.jp
}

\begin{abstract}
Riesz decomposition theorem says that a superharmonic function is locally represented as the sum of a potential and a harmonic function. In this paper we introduce a generalized Riesz kernel and study the boundary growth for its potential as an extension of Gardiner [3] in the variable settings.
\end{abstract}

\section{Introduction}

In the $N$-dimensional Euclidean space $\mathbf{R}^{N}$, we use the notation $B(x, r)$ to denote the open ball centered at $x$ of radius $r$, whose boundary is written as $S(x, r)$. Set $\mathbf{B}=B(0,1)$. The spherical mean of $u$ over $S(0, r)$ is defined by

$$
M(u, r)=\frac{1}{|S(0, r)|} \int_{S(0, r)} u(x) d S(x),
$$

where $|S(0, r)|=\omega_{N-1} r^{N-1}$ with $\omega_{N-1}$ denoting the area of the unit sphere and $d S$ denotes the surface area measure on $S(0,1)$. It is known that if $u$ is superharmonic in $\mathbf{B}$, then $M(u, r)$ is nonincreasing. If $u$ is superharmonic in $\mathbf{B}$ and $\lim _{r \rightarrow 1} M(u, r)>-\infty$, then $u$ is represented as the sum of the Green potential and a harmonic function :

$$
u(x)=\int_{\mathbf{B}} G(x, y) d \mu(y)+\text { a harmonic function; }
$$

see Theorem 2.3 and Remark 2.4 below. For this, let us consider a generalized Riesz kernel

$$
K_{\alpha, m}(x, y)=\frac{1}{(N-\alpha) \omega_{N-1}} \times\left\{\begin{array}{lc}
I_{\alpha}(x-y) & \text { when } y \in B(0,1 / 2), \\
I_{\alpha}(x-y)-\sum_{\ell=0}^{m}\left(1-|y|^{2}\right)^{\ell} \phi_{\alpha, \ell}\left(x, y^{*}\right) & \text { when } y \in \mathbf{B} \backslash B(0,1 / 2),
\end{array}\right.
$$

where $I_{\alpha}(x-y)=|x-y|^{\alpha-N}(0<\alpha<N)$ and $m \geq 0$ (see Section 2 for the definition of $\left.\phi_{\alpha, \ell}\left(x, y^{*}\right)\right)$.

https://doi.org/10.5186/aasfm.2019.4403

2010 Mathematics Subject Classification: Primary 31B15, 46E35.

Key words: Variable exponent, spherical means, superharmonic functions, generalized Riesz potentials. 
The $L^{q}(1 \leq q<\infty)$ mean over the spherical surface $S(0, r)$ for a function $u$ is defined by

$$
S_{q}(u, r)=\left(\frac{1}{|S(0, r)|} \int_{S(0, r)}|u(x)|^{q} d S(x)\right)^{1 / q}=\left(\frac{1}{\omega_{N-1}} \int_{S(0,1)}|u(r \sigma)|^{q} d S(\sigma)\right)^{1 / q} .
$$

Our starting point is a result by Gardiner [3, Theorem 2] which states that when $(N-3) /(N-1)<1 / q \leq(N-2) /(N-1)$ and $q \geq 1$,

$$
\liminf _{r \rightarrow 1-}(1-r)^{N-1-(N-1) / q} S_{q}(G \mu, r)=0
$$

for a Green potential $G \mu$ on the unit ball B. We refer the reader to [10] for the plane case and [4, Sect. 5] for versions of Gardiner's result for Riesz potentials. Moreover, in [8], the first and the third authors studied the existence of boundary limits for BLD (Beppo Levi and Deny) functions $u$ on the unit ball $\mathbf{B}$ of $\mathbf{R}^{N}$ satisfying

$$
\int_{\mathbf{B}}|\nabla u(x)|^{p}(1-|x|)^{\gamma} d x<\infty
$$

where $\nabla$ denotes the gradient, $1<p<\infty$ and $-1<\gamma<p-1$. More precisely, it was shown that

$$
\liminf _{r \rightarrow 1-}(1-r)^{(N-p+\gamma) / p-(N-1) / q} S_{q}(u, r)=0
$$

when $q>0$ and $(N-p-1) /(p(N-1))<1 / q<(N-p+\gamma) /(p(N-1))$.

Set

$$
C(0, r)=\mathbf{B} \backslash B(0, r)
$$

for $0<r<1$. For $m \geq 0$, denote by $M^{p(\cdot), m, \omega}(\mathbf{B})$ the family of all functions $f \in L_{\text {loc }}^{1}(\mathbf{B})$ such that

$$
\|f\|_{M^{p(\cdot), m, \omega}(\mathbf{B})}=\sup _{0<r<1} \omega(1-r)\|f\|_{L^{p(\cdot), m}(C(0, r))}<\infty
$$

with a variable exponent $p(\cdot)$ (see Section 3 ). In connection with Gardiner's result [3] and [8, Theorem 1], our main aim in this paper is to discuss the weighted limit:

$$
\liminf _{r \rightarrow 1-}(1-r)^{d} \omega(1-r)^{p} S_{q}\left(\left|K_{\alpha, m} f\right|^{p(r)}, r\right)
$$

for $f \in M^{p(\cdot), m+1, \omega}(\mathbf{B})$, where the exponent $d$ will be given later and

$$
K_{\alpha, m} f(x)=\int_{\mathbf{B}} K_{\alpha, m}(x, y) f(y) d y
$$

(see Theorem 4.6 below). For Riesz potentials $K_{\alpha,-1} f(x)=\int_{\mathbf{B}} I_{\alpha}(x-y) f(y) d y$, we refer to [6].

For further related results on spherical means, see e.g. [5], [7] and [9].

\section{Generalized Riesz kernels}

Throughout this paper, let $C$ denote various positive constants independent of the variables in question. The symbol $g \sim h$ means that $C^{-1} h \leq g \leq C h$ for some constant $C>0$.

Write

$$
|x-y|^{2}=\left|x-y^{*}+t y^{*}\right|^{2}=\left|x-y^{*}\right|^{2}\left(1+s /\left|x-y^{*}\right|^{2}\right),
$$

where $t=1-|y|^{2}, y^{*}=y /|y|^{2}$ and $s=t^{2}\left|y^{*}\right|^{2}+2 t\left(x-y^{*}\right) \cdot y^{*}$. Note that

$$
(1+a+b)^{\gamma}=\sum_{j=0}^{\infty}\left(\begin{array}{l}
\gamma \\
j
\end{array}\right)(a+b)^{j}=\sum_{j=0}^{\infty} \sum_{k=0}^{j}\left(\begin{array}{l}
\gamma \\
j
\end{array}\right)\left(\begin{array}{l}
j \\
k
\end{array}\right) a^{k} b^{j-k}
$$


The double series converges absolutely when $|a|+|b|<1$. Hence we have the following lemma.

Lemma 2.1. [1, Lemma 2.1] Let $x, y \in \mathbf{R}^{N}$ and $t \in \mathbf{R}$. If $|t|\left|y^{*}\right|<(\sqrt{2}-1) \mid x-$ $y^{*} \mid$, then

$$
\begin{aligned}
\left|x-y^{*}+t y^{*}\right|^{\alpha-N} & =\sum_{\ell=0}^{\infty}\left(\sum_{\ell / 2 \leq j \leq \ell} a_{\ell, j}\left|x-y^{*}\right|^{\alpha-N-2 j}\left(x \cdot y^{*}-\left|y^{*}\right|^{2}\right)^{2 j-\ell}\left|y^{*}\right|^{2(\ell-j)}\right) t^{\ell} \\
& =\sum_{\ell=0}^{\infty} \phi_{\alpha, \ell}\left(x, y^{*}\right) t^{\ell}
\end{aligned}
$$

where

$$
\phi_{\alpha, \ell}\left(x, y^{*}\right)=\sum_{\ell / 2 \leq j \leq \ell} a_{\ell, j}\left|x-y^{*}\right|^{\alpha-N-2 j}\left(x \cdot y^{*}-\left|y^{*}\right|^{2}\right)^{2 j-\ell}\left|y^{*}\right|^{2(\ell-j)}
$$

and

$$
a_{\ell, j}=\left(\begin{array}{c}
(\alpha-N) / 2 \\
j
\end{array}\right)\left(\begin{array}{c}
j \\
\ell-j
\end{array}\right) 2^{2 j-\ell} .
$$

In what follows, let $m \geq 0$. Now let us define

$$
K_{\alpha, m}(x, y)=c(\alpha, N) \times\left\{\begin{array}{lc}
|x-y|^{\alpha-N} & \text { when } y \in B(0,1 / 2), \\
|x-y|^{\alpha-N}-\sum_{\ell=0}^{m}\left(1-|y|^{2}\right)^{\ell} \phi_{\alpha, \ell}\left(x, y^{*}\right) & \text { when } y \in \mathbf{B} \backslash B(0,1 / 2),
\end{array}\right.
$$

where $c(\alpha, N)=1 /\left((N-\alpha) \omega_{N-1}\right)$.

Lemma 2.2. (cf. [1, Lemma 2.2])

(1) For $y \in \mathbf{B}$ and $N>2, \Delta K_{2, m}(\cdot, y)=\delta_{y}$ on $\mathbf{B}$;

(2) there exists a constant $C>0$ such that

$$
\left|K_{\alpha, m}(x, y)\right| \leq C|x-y|^{\alpha-N}
$$

for all $x, y \in \mathbf{B}$;

(3) there exists a constant $C>0$ such that

$$
\left|K_{\alpha, m}(x, y)\right| \leq C|x-y|^{\alpha-N-m-1}(1-|y|)^{m+1}
$$

for all $x, y \in \mathbf{B}$.

Proof. First we show assertion (1). Consider $F_{\alpha}(t)=\left|x-y^{*}+t y^{*}\right|^{\alpha-N}$. Then

$$
\phi_{2, \ell}\left(x, y^{*}\right)=F_{2}^{(\ell)}(0) / \ell !,
$$

so that $\phi_{2, \ell}\left(\cdot, y^{*}\right)$ is harmonic in $\mathbf{B}$. Thus (1) follows.

Next we show assertion (2). We may assume $y \in \mathbf{B} \backslash B(0,1 / 2)$. Note that

$$
\begin{aligned}
\left|\phi_{\alpha, \ell}\left(x, y^{*}\right)\right| & \leq\left.\left.\sum_{\ell / 2 \leq j \leq \ell}\left|a_{\ell, j}\right|\left|x-y^{*}\right|^{\alpha-N-2 j}\left|x \cdot y^{*}-\right| y^{*}\right|^{2}\right|^{2 j-\ell}\left|y^{*}\right|^{2(\ell-j)} \\
& =\sum_{\ell / 2 \leq j \leq \ell}\left|a_{\ell, j}\right|\left|x-y^{*}\right|^{\alpha-N-2 j}\left|x \cdot y^{*} /\right| y^{*}|-| y^{*}||^{2 j-\ell}\left|y^{*}\right|^{\ell} \\
& =C\left|x-y^{*}\right|^{\alpha-N-\ell}\left|y^{*}\right|^{\ell}
\end{aligned}
$$


so that

$$
\left|\phi_{\alpha, \ell}\left(x, y^{*}\right)\right|\left(1-|y|^{2}\right)^{\ell} \leq C\left|x-y^{*}\right|^{\alpha-N-\ell}\left|y^{*}\right|^{\ell}\left(1-|y|^{2}\right)^{\ell} \leq C\left|x-y^{*}\right|^{\alpha-N}
$$

since

Hence we obtain

$$
\frac{\left|x-y^{*}\right|}{1-|y|} \geq \frac{\left|y^{*}\right|-|x|}{1-|y|} \geq \frac{\left|y^{*}\right|-1}{1-|y|}=\left|y^{*}\right|
$$

$$
\left|K_{\alpha, m}(x, y)\right| \leq C\left(|x-y|^{\alpha-N}+\left|x-y^{*}\right|^{\alpha-N}\right) \leq C|x-y|^{\alpha-N}
$$

since

$$
\left|x-y^{*}\right|>|y|\left|x-y^{*}\right|=|x|\left|x^{*}-y\right|=\left(|x-y|^{2}+\left(1-|x|^{2}\right)\left(1-|y|^{2}\right)\right)^{1 / 2}>|x-y| .
$$

Finally, we show assertion (3). If $1-|y|^{2} \geq|x-y| / 4$, then

$$
\left|K_{\alpha, m}(x, y)\right| \leq C|x-y|^{\alpha-N} \leq C|x-y|^{\alpha-N-m-1}(1-|y|)^{m+1} .
$$

Hence we show the case $1-|y|^{2}<|x-y| / 4$ and $1 / 2 \leq|y|<1$. By Taylor's theorem, one can find $0<\theta<1$ such that

$$
K_{\alpha, m}(x, y)=\frac{1}{(N-\alpha) \sigma_{N}(m+1) !} F_{\alpha}^{(m+1)}\left(\theta\left(1-|y|^{2}\right)\right)\left(1-|y|^{2}\right)^{m+1} .
$$

Set

$$
\begin{aligned}
G(S) & =(1+S)^{(\alpha-N) / 2} \\
S & =S(t)=\frac{2 t\left(x-y^{*}\right) \cdot y^{*}}{\left|x-y^{*}\right|^{2}}+\frac{t^{2}\left|y^{*}\right|^{2}}{\left|x-y^{*}\right|^{2}}
\end{aligned}
$$

and

$$
H(t)=G(S(t))
$$

Then we see by induction on $m$ that $H^{(m+1)}(t)$ is of the form

$$
H^{(m+1)}(t)=\sum_{0 \leq \ell \leq(m+1) / 2} c_{m ; \ell} G^{(m+1-\ell)}(S(t))\left(S^{(1)}(t)\right)^{m+1-2 \ell}\left(S^{(2)}(t)\right)^{\ell},
$$

where $c_{m ; \ell}$ are constants. Here note that in case $0 \leq t \leq 1-|y|^{2} \leq|x-y| / 4$,

$$
\begin{aligned}
|x-y| & \leq\left|x-y^{*}\right| \leq|x-y|+\left|y-y^{*}\right| \leq 3|x-y| / 2, \\
\left|x-y^{*}+t y^{*}\right| & \geq\left|x-y^{*}\right|-t\left|y^{*}\right| \geq|x-y| / 2
\end{aligned}
$$

and hence

Thus

$$
-\frac{8}{9} \leq \frac{(|x-y| / 2)^{2}-\left|x-y^{*}\right|^{2}}{\left|x-y^{*}\right|^{2}} \leq S(t) \leq 0
$$

$$
\left|S^{(1)}(t)\right| \leq \frac{2\left|y^{*}\right|}{\left|x-y^{*}\right|}+\frac{2\left(1-|y|^{2}\right)\left|y^{*}\right|^{2}}{\left|x-y^{*}\right|^{2}} \leq C|x-y|^{-1}
$$

and

$$
\left|H^{(m+1)}(t)\right| \leq C \sum_{0 \leq \ell \leq(m+1) / 2}|x-y|^{-(m+1-2 \ell)}|x-y|^{-2 \ell} \leq C|x-y|^{-(m+1)}
$$

when $1-|y|^{2}<|x-y| / 4$ and $1 / 2 \leq|y|<1$. Now we obtain

$$
\begin{aligned}
\left|K_{\alpha, m}(x, y)\right| & \leq C\left|x-y^{*}\right|^{\alpha-N}\left|H^{(m+1)}\left(\theta\left(1-|y|^{2}\right)\right)\right|\left(1-|y|^{2}\right)^{m+1} \\
& \leq C|x-y|^{(\alpha-N)-(m+1)}\left(1-|y|^{2}\right)^{m+1}
\end{aligned}
$$

which proves the result. 
For reader's convenience we show Riesz decomposition theorem in the following.

Theorem 2.3. [1, Theorem 5.5] Let $u$ be superharmonic in $\mathbf{B}$.

(1) If

$$
\lim _{r \rightarrow 1} M(u, r)>-\infty
$$

then

where $h$ is harmonic in $\mathbf{B}$.

$$
u(x)=\int_{\mathbf{B}} K_{2,0}(x, y) d \mu(y)+h(x),
$$

(2) If

$$
\liminf _{r \rightarrow 1}(1-r)^{a} M(u, r)>-\infty
$$

for some $a>0$, then

$$
u(x)=\int_{\mathbf{B}} K_{2, m}(x, y) d \mu(y)+h_{0}(x),
$$

where $h_{0}$ is harmonic in $\mathbf{B}$ and $m$ is an integer greater than $a$.

Remark 2.4. Note that

$$
K_{2,0}(x, y)=G(x, y)+\left(|y|^{2-N}-1\right)\left|x-y^{*}\right|^{2-N} .
$$

If $u$ is superharmonic in $\mathbf{B}$ and

$$
\lim _{r \rightarrow 1} M(u, r)>-\infty
$$

then

$$
u(x)=\int_{\mathbf{B}} G(x, y) d \mu(y)+v(x)=\int_{\mathbf{B}} K_{2,0}(x, y) d \mu(y)+h(x),
$$

where $v$ and $h$ are harmonic in $\mathbf{B}$.

\section{Variable exponent on the unit ball}

Let $p(\cdot)$ be a variable exponent on $\mathbf{B}$ such that

$(p 1) 1 \leq p^{-} \equiv \inf _{x \in \mathbf{B}} p(x) \leq \sup _{x \in \mathbf{B}} p(x) \equiv p^{+}<\infty$;

$(p 2)|p(x)-p(y)| \leq \frac{c_{\mathbf{B}}}{\log (e /|| x|-| y||)}$ for $x, y \in \mathbf{B}$ with a constant $c_{\mathbf{B}}>0$.

By (p2), we see that $p(\cdot)$ is uniformly continuous on $\mathbf{B}$ and a radial function on $\mathbf{B}$. Thus we have

(p3) there exists a constant $p \geq 1$ such that

$$
|p(x)-p| \leq \frac{c_{\mathbf{B}}}{\log (e /(1-|x|))} \quad \text { for } x \in \mathbf{B} .
$$

For simplicity, we set $p(r)=p(x)$ with $r=|x|$. A typical example of $p(\cdot)$ is of the form

$$
p(x)=p+\frac{c}{\log (e /(1-|x|))}
$$

as in [2].

Let $\Omega$ be a measurable set in $\mathbf{B}$. For $m \geq 0$, the variable exponent Lebesgue spaces

$$
L^{p(\cdot), m}(\Omega)=\left\{f \in L_{\mathrm{loc}}^{1}(\Omega) ; \int_{\Omega}\left((1-|y|)^{m}|f(y)|\right)^{p(y)} d y<\infty\right\}
$$


is a Banach space with respect to the norm

$$
\|f\|_{L^{p(\cdot), m}(\Omega)}=\inf \left\{\lambda>0 ; \int_{\Omega}\left(\frac{(1-|y|)^{m}|f(y)|}{\lambda}\right)^{p(y)} d y \leq 1\right\} .
$$

Further we consider a weight $\omega$ such that

$(\omega 1) \omega(r)>0$ for $0<r \leq 1$;

$(\omega 2) \omega$ is almost decreasing in $(0,1]$, that is, there is a constant $C>0$ such that

$$
\omega(t) \leq C \omega(s) \text { when } 0<s<t \leq 1
$$

$(\omega 3) \omega$ is doubling on $(0,1]$.

Throughout this paper, we always assume that $\omega$ satisfies all of $(\omega 1)-(\omega 3)$. We see that $\omega(r)=r^{-\nu}\left(\log \left(e+r^{-1}\right)\right)^{\tau}$ is almost decreasing when $\nu>0$ and $\tau \in \mathbf{R}$. Set

$$
C(0, r)=\mathbf{B} \backslash B(0, r)
$$

for $0<r<1$. For $m \geq 0$, denote by $M^{p(\cdot), m, \omega}(\mathbf{B})$ the family of all functions $f \in L_{\mathrm{loc}}^{1}(\mathbf{B})$ such that

$$
\|f\|_{M^{p(\cdot), m, \omega}(\mathbf{B})}=\sup _{0<r<1} \omega(1-r)\|f\|_{L^{p(\cdot), m}(C(0, r))}<\infty .
$$

Let us begin with the following elementary estimates for spherical means.

Lemma 3.1. [6, Lemma 2.1] Let $0<a<1$ and $c_{1}$ be positive constants. If $y \in \mathbf{B}$ and $1 / 2<t<\min \left\{1, c_{1}|y|\right\}$, then there exists a constant $C>0$ such that

$$
\int_{S(0,1)}|t \sigma-y|^{a-N} d S(\sigma) \leq C|t-| y||^{a-1}
$$

For later use, we need a version of Lemma 3.1 when $a>1$.

Lemma 3.2. [6, Lemma 2.2] Let $1<a<N$ and $c_{1}$ be positive constants. If $y \in \mathbf{B}$ and $1 / 2<t<\min \left\{1, c_{1}|y|\right\}$, then there exists a constant $C>0$ such that

$$
\int_{\{\sigma \in S(0,1):|t \sigma-y|<1-t\}}|t \sigma-y|^{a-N} d S(\sigma) \leq C(1-t)^{a-1} .
$$

Set

$$
I=\frac{1}{|B(x, t)|} \int_{B(x, t) \cap \mathbf{B}}|f(y)| d y
$$

and

$$
J=\left(\frac{1}{|B(x, t)|} \int_{B(x, t) \cap \mathbf{B}}|f(y)|^{p(y)} d y\right)^{1 / p(x)},
$$

where $|B(x, t)|$ denotes the volume of balls $B(x, t)$. Then $I$ is estimated by $J$ as follows.

Lemma 3.3. [6, Lemma 2.4] Let $\gamma>0$. If $J \leq \beta_{1} t^{-\beta_{2}}$ for some constants $\beta_{1}, \beta_{2}>0$, then there exists a constant $C>0$ such that

$$
I \leq C\left(t^{\gamma}+J\right)
$$

for all $x \in \mathbf{B}, 0<t<1$ and $f \in L_{\text {loc }}^{1}(\mathbf{B})$, where a constant $C$ depends only on $\beta_{1}, \beta_{2}$, $\gamma$ and $c_{1}$.

Finally it is convenient to see the following estimates.

Lemma 3.4. [6, Lemma 2.5] For $1 / 2<r<1$,

$$
\omega(1-r)^{p(r)} \sim \omega(1-r)^{p}, \quad(1-r)^{p(r)} \sim(1-r)^{p} .
$$




\section{Spherical means near the boundary}

In what follows we prepare several estimates for Riesz potentials of functions in $M^{p(\cdot), m+1, \omega}(\mathbf{B})$. For this purpose, write

$$
\begin{aligned}
K_{\alpha, m} f(x)= & \int_{B(x,(1-|x|) / 2)} K_{\alpha, m}(x, y) f(y) d y \\
& +\int_{\{y \in \mathbf{B} \backslash B(x,(1-|x|) / 2): 1-|y| \leq 1-|x|\}} K_{\alpha, m}(x, y) f(y) d y \\
& +\int_{\{y \in \mathbf{B} \backslash B(x,(1-|x|) / 2): 1-|y|>1-|x|\}} K_{\alpha, m}(x, y) f(y) d y \\
= & K_{1}(x)+K_{2}(x)+K_{3}(x) .
\end{aligned}
$$

We first give an estimate for $K_{1}(x)$. For this note by Lemma $2.2(2)$

$$
\left|K_{1}(x)\right| \leq C \int_{B(x,(1-|x|) / 2)}|x-y|^{\alpha-N} f(y) d y .
$$

Set

$$
A(0, r)=B(0, r+(1-r) / 2) \backslash B(0, r-(1-r) / 2)
$$

for $1 / 2<r<1$.

Lemma 4.1. Let $1 \leq q<\infty$.

(1) Let $\beta>0$. Suppose

$$
(N-1) / q \leq N-\alpha p
$$

Then, for $\varepsilon>0$, there exist constants $C>0$ and $1 / 2<r_{1}<1$ such that

$$
\begin{aligned}
& S_{q}\left(\left|K_{1}\right|^{p(r)}, r\right) \leq C \omega(1-r)^{-p}\left\{(1-r)^{\beta}+(1-r)^{\varepsilon(2 p-1)-(m+1) p}\right. \\
& \left.\times\left.\int_{A(0, r)}|r-| y\right|^{\alpha p(r)-\varepsilon(2 p(r)-1)-N+(N-1) / q}\left(\omega(1-|y|)(1-|y|)^{m+1} f(y)\right)^{p(y)} d y\right\}
\end{aligned}
$$

for all $r_{1}<r<1$ and nonnegative measurable functions $f$ on $\mathbf{B}$ with $\|f\|_{M^{p(\cdot), m+1, \omega}(\mathbf{B})} \leq 1$.

(2) Suppose

$$
(N-1) / q>N-\alpha p .
$$

Then there exist constants $C>0$ and $1 / 2<r_{1}<1$ such that

$$
S_{q}\left(\left|K_{1}\right|^{p(r)}, r\right) \leq C(1-r)^{(\alpha-m-1) p-N+(N-1) / q} \omega(1-r)^{-p}
$$

for all $r_{1}<r<1$ and nonnegative measurable functions $f$ on $\mathbf{B}$ with $\|f\|_{M^{p(\cdot), m+1, \omega}(\mathbf{B})} \leq 1$.

Proof. Let $f$ be a nonnegative measurable function on $\mathbf{B}$ with $\|f\|_{M^{p(\cdot), m+1, \omega}(\mathbf{B})} \leq$ 1 and let $1 / 2<r=|x|<1$. First we show the assertion (1). Let $\beta>0$ and let $\varepsilon>0$ such that

$$
(N-1) / q<N-\alpha p+\varepsilon(2 p-1)
$$


We have

$$
\begin{aligned}
\left|K_{1}(x)\right| & \leq C \int_{B(x,(1-r) / 2)}|x-y|^{\alpha-N} f(y) d y \\
& \leq C \int_{0}^{1-r}\left(\frac{1}{|B(x, t)|} \int_{B(x, t) \cap A(0, r)} f(y) d y\right) t^{\alpha-1} d t \\
& \leq C(1-r)^{\varepsilon} \int_{0}^{1-r}\left(\frac{1}{|B(x, t)|} \int_{B(x, t) \cap A(0, r)} t^{\alpha-2 \varepsilon} f(y) d y\right) t^{\varepsilon-1} d t
\end{aligned}
$$

since $B(x,(1-r) / 2) \subset A(0, r)$. Take $1 / 2<r_{1}<1$ such that

$$
C_{1, p}=\sup _{r_{1}<r<1}\{\alpha p(r)-\varepsilon(2 p(r)-1)-N+(N-1) / q\}<0 .
$$

Letting $s=r-(1-r) / 2$, we see that

$$
\begin{aligned}
& \int_{A(0, r)}\left(\omega(1-r)(1-|y|)^{m+1} f(y)\right)^{p(y)} d y \\
& \leq \int_{C(0, s)}\left(\omega(2(1-s) / 3)(1-|y|)^{m+1} f(y)\right)^{p(y)} d y \\
& \leq C \int_{C(0, s)}\left(\omega(1-s)(1-|y|)^{m+1} f(y)\right)^{p(y)} d y \leq C,
\end{aligned}
$$

so that

$$
\int_{A(0, r)}\left(\omega(1-r)(1-|y|)^{m+1} f(y)\right)^{p(y)} d y \leq C .
$$

Note here from (4.1) that

$$
\left(\frac{1}{|B(x, t)|} \int_{B(x, t) \cap A(0, r)}\left(\omega(1-r)(1-|y|)^{m+1} f(y)\right)^{p(y)} d y\right)^{1 / p(r)} \leq C t^{-N / p^{-}}
$$

for $0<t<1$.

Take $\gamma>0$ such that

$$
\gamma>\max \left\{\varepsilon\left(2-1 / p^{+}\right)-\alpha, \beta / p-\alpha+m+1\right\} .
$$

By Jensen's inequality and Lemmas 3.3 and 3.4, we have

$$
\begin{aligned}
(\omega & \left.(1-r)(1-r)^{-2 \varepsilon}\left|K_{1}(x)\right|\right)^{p(r)} \\
\leq & C\left(\omega(1-r)(1-r)^{-\varepsilon-m-1}\right. \\
& \left.\times \int_{0}^{1-r}\left(\frac{1}{|B(x, t)|} \int_{B(x, t) \cap A(0, r)} t^{\alpha-2 \varepsilon}(1-|y|)^{m+1} f(y) d y\right) t^{\varepsilon-1} d t\right)^{p(r)} \\
\leq & C(1-r)^{-\varepsilon-(m+1) p} \\
& \times \int_{0}^{1-r}\left(\frac{1}{|B(x, t)|} \int_{B(x, t) \cap A(0, r)} t^{\alpha-2 \varepsilon} \omega(1-r)(1-|y|)^{m+1} f(y) d y\right)^{p(r)} t^{\varepsilon-1} d t \\
= & C(1-r)^{-\varepsilon-(m+1) p} \\
& \times \int_{0}^{1-r} t^{(\alpha-2 \varepsilon) p(r)}\left(\frac{1}{|B(x, t)|} \int_{B(x, t) \cap A(0, r)} \omega(1-r)(1-|y|)^{m+1} f(y) d y\right)^{p(r)} t^{\varepsilon-1} d t
\end{aligned}
$$




$$
\begin{aligned}
\leq & C(1-r)^{-\varepsilon-(m+1) p}\left\{\int_{0}^{1-r} t^{\alpha p(r)-\varepsilon(2 p(r)-1)+\gamma p(r)-1} d t\right. \\
& \left.+\int_{0}^{1-r} t^{(\alpha-2 \varepsilon) p(r)-N}\left(\int_{B(x, t) \cap A(0, r)}\left(\omega(1-r)(1-|y|)^{m+1} f(y)\right)^{p(y)} d y\right) t^{\varepsilon-1} d t\right\} \\
\leq & C\left\{(1-r)^{(\alpha-m-1+\gamma) p-2 \varepsilon p}+(1-r)^{-\varepsilon-(m+1) p}\right. \\
& \left.\times \int_{0}^{1-r} t^{(\alpha-2 \varepsilon) p(r)-N}\left(\int_{B(x, t) \cap A(0, r)}\left(\omega(1-r)(1-|y|)^{m+1} f(y)\right)^{p(y)} d y\right) t^{\varepsilon-1} d t\right\} \\
\leq & C\left\{(1-r)^{\beta-2 \varepsilon p}\right. \\
& \left.+(1-r)^{-\varepsilon-(m+1) p} \int_{A(0, r)}|x-y|^{\alpha p(r)-\varepsilon(2 p(r)-1)-N}\left(\omega(1-|y|)(1-|y|)^{m+1} f(y)\right)^{p(y)} d y\right\}
\end{aligned}
$$

for $r_{1}<r<1$, since

$$
\alpha p(r)-\varepsilon(2 p(r)-1)-N<-(N-1) / q+C_{1, p}<0
$$

for $r_{1}<r<1$. Then Minkowski's inequality and Lemma 3.1 yield

$$
\begin{aligned}
& S_{q}\left(\left|K_{1}\right|^{p(r)}, r\right) \\
& \leq C \omega(1-r)^{-p}\left\{(1-r)^{\beta}+(1-r)^{\varepsilon(2 p-1)-(m+1) p}\right. \\
& \left.\quad \times \int_{A(0, r)} S_{q}\left(|\cdot-y|^{\alpha p(r)-\varepsilon(2 p(r)-1)-N}, r\right)\left(\omega(1-|y|)(1-|y|)^{m+1} f(y)\right)^{p(y)} d y\right\} \\
& \leq C \omega(1-r)^{-p}\left\{(1-r)^{\beta}+(1-r)^{\varepsilon(2 p-1)-(m+1) p}\right. \\
& \left.\quad \times\left.\int_{A(0, r)}|r-| y\right|^{\alpha p(r)-\varepsilon(2 p(r)-1)-N+(N-1) / q}\left(\omega(1-|y|)(1-|y|)^{m+1} f(y)\right)^{p(y)} d y\right\}
\end{aligned}
$$

for $r_{1}<r<1$, since $r \sim|y|$ on $A(0, r)$ and

$$
\alpha p(r)-\varepsilon(2 p(r)-1)-N+(N-1) / q \leq C_{1, p}<0
$$

for $r_{1}<r<1$. Thus assertion (1) is proved.

Next we shall show assertion (2). Let $\varepsilon>0$ such that

$$
(N-1) / q>N-\alpha p+\varepsilon(p-1)>0 .
$$

Take $1 / 2<r_{1}<1$ such that

$$
\begin{aligned}
& \inf _{r_{1}<r<1}\{\alpha p(r)-\varepsilon(p(r)-1)-N+(N-1) / q\}>0, \\
& \sup _{r_{1}<r<1}\{\alpha p(r)-\varepsilon(p(r)-1)-N\}<0
\end{aligned}
$$

and $\gamma>0$ such that

$$
\gamma>\varepsilon\left(1-1 / p^{+}\right)-\alpha .
$$


As in the above considerations, we obtain by Lemma 3.2

$$
\begin{aligned}
& S_{q}\left(\left(\omega(1-r)(1-r)^{-\varepsilon}\left|K_{1}\right|\right)^{p(r)}, r\right) \\
& \leq C(1-r)^{-\varepsilon-(m+1) p}\left\{(1-r)^{\alpha p-\varepsilon(p-1)+\gamma p}\right. \\
& \left.\quad+\int_{A(0, r)} S_{q}\left(|\cdot-y|^{\alpha p(r)-\varepsilon(p(r)-1)-N} \chi_{B(y,(1-r) / 2)}, r\right)\left(\omega(1-r)(1-|y|)^{m+1} f(y)\right)^{p(y)} d y\right\} \\
& \leq C(1-r)^{-\varepsilon-(m+1) p}\left\{(1-r)^{\alpha p-\varepsilon(p-1)+\gamma p}\right. \\
& \left.\quad+(1-r)^{\alpha p-\varepsilon(p-1)-N+(N-1) / q} \int_{C(0, r-(1-r) / 2)}\left(\omega(1-r)(1-|y|)^{m+1} f(y)\right)^{p(y)} d y\right\} \\
& \leq C(1-r)^{(\alpha-m-1-\varepsilon) p-N+(N-1) / q}
\end{aligned}
$$

for $r_{1}<r<1$. Thus assertion (2) is proved.

Let $d(\cdot)$ be a valuable exponent on $[0,1)$ such that

(d1) $0<\inf _{t \in[0,1)} d(t) \leq \sup _{t \in[0,1)} d(t)<1$;

(d2) there exists a positive constant $0<d<1$ such that

$$
|d(t)-d| \leq \frac{c_{d}}{\log (e /(1-t))} \quad \text { for } 0<t<1
$$

with a constant $c_{d}>0$.

Set

$$
G(t)=\left.(1-t)^{d} \int_{A(0, t)}|t-| y\right|^{-d(t)} g(y) d y
$$

for a nonnegative measurable function $g$.

To complete the estimate for $K_{1}$, we use the following result.

Lemma 4.2. [7, Lemma 2.7] Let $M>0$. If $\sup _{0<t<1} \int_{A(0, t)} g(y) d y \leq M$, then there exists a constant $C>0$ such that

$$
\inf _{1-2^{-j+1}<t<1-2^{-j}} G(t)<C M \quad \text { for each positive integer } j .
$$

Next we treat $K_{2}(x)$. For this note from Lemma $2.2(3)$ that

$$
\left|K_{2}(x)\right| \leq C \int_{\{y \in \mathbf{B} \backslash B(x,(1-|x|) / 2): 1-|y| \leq 1-|x|\}}|x-y|^{\alpha-N-m-1}(1-|y|)^{m+1} f(y) d y \text {. }
$$

Lemma 4.3. Let $1 \leq q<\infty$, and suppose

$$
(N-1) / q<N-(\alpha-m-1) p .
$$

Then there exists a constant $C>0$ such that

$$
S_{q}\left(\left|K_{2}\right|^{p(r)}, r\right) \leq C(1-r)^{(\alpha-m-1) p-N+(N-1) / q} \omega(1-r)^{-p}
$$

for all $1 / 2<r<1$ and nonnegative measurable functions $f$ on $\mathbf{B}$ with $\|f\|_{M^{p(\cdot), m+1, \omega}(\mathbf{B})}$ $\leq 1$.

Proof. Let $f$ be a nonnegative measurable function on $\mathbf{B}$ with $\|f\|_{M^{p(\cdot), m+1, \omega}(\mathbf{B})} \leq$ 1 and let $1 / 2<r=|x|<1$. Let $\varepsilon>0$ such that

$$
(N-1) / q<N-(\alpha-m-1) p-\varepsilon(p-1) \text {. }
$$


We have by Lemma $2.2(3)$

$$
\begin{aligned}
\left|K_{2}(x)\right| & \leq C \int_{\{y \in \mathbf{B} \backslash B(x,(1-|x|) / 2): 1-|y| \leq 1-|x|\}}|x-y|^{\alpha-N-m-1}(1-|y|)^{m+1} f(y) d y \\
& \leq C \int_{(1-r) / 2}^{2}\left(\frac{1}{|B(x, t)|} \int_{B(x, t)} f_{2, x}(y) d y\right) t^{\alpha-m-2} d t \\
& \leq C \int_{(1-r) / 2}^{2}\left(\frac{1}{|B(x, t)|} \int_{B(x, t)} t^{\alpha-m-1+\varepsilon} f_{2, x}(y) d y\right) t^{-\varepsilon-1} d t,
\end{aligned}
$$

where $f_{2, x}(y)=(1-|y|)^{m+1} f(y) \chi_{E_{2, x}}(y)$ with $E_{2, x}=\{y \in \mathbf{B} \backslash B(x,(1-r) / 2): 1-|y| \leq$ $1-r\}$ and $\chi_{E}$ is the characteristic function of $E$.

Note from (p3) that

$$
t^{p(r)}=t^{p} t^{p(r)-p} \leq C t^{p} t^{-c_{\mathbf{B}} / \log (e /(1-r))} \leq C t^{p}(1-r)^{-c_{\mathbf{B}} / \log (e /(1-r))} \leq C t^{p}
$$

and

$$
t^{p(r)} \geq C t^{p} t^{c_{\mathbf{B}} / \log (e /(1-r))} \geq C t^{p}(1-r)^{c_{\mathbf{B}} / \log (e /(1-r))} \geq C t^{p}
$$

for $(1-r) / 2<t<2$. Since

$$
\int_{B(x, t)}\left(\omega(1-r) f_{2, x}(y)\right)^{p(y)} d y \leq \int_{C(0, r)}\left(\omega(1-r)(1-|y|)^{m+1} f(y)\right)^{p(y)} d y \leq C
$$

by the fact that $E_{2, x} \subset C(0, r)$, we have

$$
\left(\frac{1}{|B(x, t)|} \int_{B(x, t)}\left(\omega(1-r) f_{2, x}(y)\right)^{p(y)} d y\right)^{1 / p(r)} \leq C t^{-N / p^{-}}
$$

for $(1-r) / 2<t<2$. We have by Jensen's inequality and Lemma 3.3 with $\gamma>$ $-\varepsilon(1-1 / p)-\alpha+m+1$

$$
\begin{aligned}
& \left(\omega(1-r)(1-r)^{\varepsilon}\left|K_{2}(x)\right|\right)^{p(r)} \\
& \leq C(1-r)^{\varepsilon} \int_{(1-r) / 2}^{2}\left(\frac{1}{|B(x, t)|} \int_{B(x, t)} t^{\alpha-m-1+\varepsilon} \omega(1-r) f_{2, x}(y) d y\right)^{p(r)} t^{-\varepsilon-1} d t \\
& \leq C(1-r)^{\varepsilon}\left\{1+\int_{(1-r) / 2}^{2} t^{(\alpha-m-1+\varepsilon) p}\left(\frac{1}{|B(x, t)|} \int_{B(x, t)}\left(\omega(1-r) f_{2, x}(y)\right)^{p(y)} d y\right) t^{-\varepsilon-1} d t\right\} \\
& \leq C(1-r)^{\varepsilon}\left\{1+\int_{\mathbf{B}}|x-y|^{(\alpha-m-1) p+\varepsilon(p-1)-N}\left(\omega(1-r) f_{2, x}(y)\right)^{p(y)} d y\right\}
\end{aligned}
$$

for $1 / 2<r<1$, since

$$
\int_{(1-r) / 2}^{2} t^{(\alpha-m-1) p+\varepsilon(p-1)+\gamma p-1} d t \leq C
$$

and

$$
(\alpha-m-1) p+\varepsilon(p-1)-N<-(N-1) / q<0 .
$$

By Lemma 3.1, we see that

$$
\begin{aligned}
& \int_{\{\sigma \in S(0,1):|t \sigma-y|>(1-t) / 2\}}|t \sigma-y|^{a-N} d S(\sigma) \\
& \leq \int_{\{\sigma \in S(0,1):|t \sigma-y|>(1-t) / 2\}}(C|(1+(1-t)) \sigma-y|)^{a-N} d S(\sigma) \\
& \leq\left. C|(1+(1-t))-| y\right|^{a-1} \leq C|1-t|^{a-1}
\end{aligned}
$$


for $1 / 2<t<1$ and $1 / 2<|y|<1$, when $a<1$. Hence Minkowski's inequality yields $S_{q}\left(\left(\omega(1-r)(1-r)^{\varepsilon}\left|K_{2}\right|\right)^{p(r)}, r\right)$

$$
\leq C(1-r)^{\varepsilon}
$$$$
\times\left\{1+\int_{\mathbf{B}} S_{q}\left(|\cdot-y|^{(\alpha-m-1) p+\varepsilon(p-1)-N} \chi_{E_{2, x}}(y), r\right)\left(\omega(1-r)(1-|y|)^{m+1} f(y)\right)^{p(y)} d y\right\}
$$$$
\leq C(1-r)^{\varepsilon}
$$

$$
\times\left\{1+(1-r)^{(\alpha-m-1) p+\varepsilon(p-1)-N+(N-1) / q} \int_{C(0, r)}\left(\omega(1-r)(1-|y|)^{m+1} f(y)\right)^{p(y)} d y\right\}
$$

$\leq C(1-r)^{(\alpha-m-1+\varepsilon) p-N+(N-1) / q}$

for $1 / 2<r<1$, since

$$
(\alpha-m-1) p+\varepsilon(p-1)-N+(N-1) / q<0 .
$$

Thus the assertion is proved.

Finally we treat $K_{3}(x)$. Note from Lemma $2.2(3)$ that

$$
\left|K_{3}(x)\right| \leq C \int_{\{y \in \mathbf{B} \backslash B(x,(1-|x|) / 2): 1-|y|>1-|x|\}}|x-y|^{\alpha-N-m-1}(1-|y|)^{m+1} f(y) d y .
$$

Lemma 4.4. Let $1 \leq q<\infty$, and suppose

$(\omega 4) t^{(\alpha-m-1) p+\varepsilon_{0}-N+(N-1) / q} \omega(t)^{-p}$ is almost decreasing on $(0,1]$ for some $\varepsilon_{0}>0$.

Then there exists a constant $C>0$ such that

$$
S_{q}\left(\left|K_{3}\right|^{p(r)}, r\right) \leq C(1-r)^{(\alpha-m-1) p-N+(N-1) / q} \omega(1-r)^{-p}
$$

for all $1 / 2<r<1$ and nonnegative measurable functions $f$ on $\mathbf{B}$ with $\|f\|_{M^{p(\cdot), m+1, \omega}(\mathbf{B})}$ $\leq 1$.

Remark 4.5. If $(\omega 4)$ holds, then

$$
(\alpha-m-1) p-N+(N-1) / q<0 .
$$

Proof of Lemma 4.4. Let $f$ be a nonnegative measurable function on $\mathbf{B}$ with $\|f\|_{M^{p(\cdot), m+1, \omega}(\mathbf{B})} \leq 1$ and let $1 / 2<r=|x|<1$. Note that $t^{p(r)} \sim t^{p}$ for $c(1-r)<$ $t<2$.

Let $\varepsilon>0$ and $\varepsilon(p-1)<\varepsilon_{0}$. Note from $(\omega 4)$ that $t^{(\alpha-m-1) p+\varepsilon(p-1)-N+(N-1) / q} \omega(t)^{-p}$ is almost decreasing on $(0,1]$ and

$$
(N-1) / q<N-(\alpha-m-1) p-\varepsilon(p-1) .
$$

We see that

$$
\int_{B(0,1 / 4)}|x-y|^{\alpha-N-m-1}(1-|y|)^{m+1} f(y) d y \leq C \int_{B(0,1 / 4)}(1-|y|)^{m+1} f(y) d y \leq C
$$

since $\|f\|_{L^{p(\cdot), m+1}(\mathbf{B})} \leq \omega(1)^{-1} \leq C$. As in the proof of Lemma 4.3, we have

$$
\begin{aligned}
\left|K_{3}(x)\right| & \leq C\left\{1+\int_{\mathbf{B}}|x-y|^{\alpha-N-m-1} f_{3, x}(y) d y\right\} \\
& \leq C\left\{1+\int_{(1-r) / 2}^{2}\left(\frac{1}{|B(x, t)|} \int_{B(x, t)} f_{3, x}(y) d y\right) t^{\alpha-m-2} d t\right\} \\
& \leq C\left\{1+\int_{(1-r) / 2}^{2}\left(\frac{1}{|B(x, t)|} \int_{B(x, t)} t^{\alpha-m-1+\varepsilon} f_{3, x}(y) d y\right) t^{-\varepsilon-1} d t\right\},
\end{aligned}
$$


where $f_{3, x}(y)=(1-|y|)^{m+1} f(y) \chi_{E_{3, x}}(y)$ with

$$
E_{3, x}=\{y \in \mathbf{B} \backslash(B(0,1 / 4) \cup B(x,(1-r) / 2)): 1-|y|>1-r\} .
$$

Since $\|f\|_{L^{p(\cdot), m+1}(\mathbf{B})} \leq C$, we have

$$
\left(\frac{1}{|B(x, t)|} \int_{B(x, t)} f_{3, x}(y)^{p(y)} d y\right)^{1 / p(r)} \leq C t^{-N / p^{-}}
$$

for $(1-r) / 2<t<2$. Since $r \sim|y|$ for $y \in \mathbf{B} \backslash B(0,1 / 4)$, in the same way as in the proof of Lemma 4.3, we see that

$$
\begin{aligned}
& \left((1-r)^{\varepsilon}\left|K_{3}(x)\right|\right)^{p(r)} \\
& \leq C\left\{(1-r)^{\varepsilon}\left(1+\int_{(1-r) / 2}^{2}\left(\frac{1}{|B(x, t)|} \int_{B(x, t)} t^{\alpha-m-1+\varepsilon} f_{3, x}(y) d y\right) t^{-\varepsilon-1} d t\right)\right\}^{p(r)} \\
& \leq C(1-r)^{\varepsilon}\left\{1+\int_{\mathbf{B}}|x-y|^{(\alpha-m-1) p+\varepsilon(p-1)-N} f_{3, x}(y)^{p(y)} d y\right\}
\end{aligned}
$$

for $1 / 2<r<1$, so that

$$
\begin{aligned}
& S_{q}\left(\left((1-r)^{\varepsilon}\left|K_{3}\right|\right)^{p(r)}, r\right) \\
& \leq C(1-r)^{\varepsilon}\left(1+\int_{\mathbf{B}} S_{q}\left(|\cdot-y|^{(\alpha-m-1) p+\varepsilon(p-1)-N} \chi_{E_{3, x}}(y), r\right) f_{3, x}(y)^{p(y)} d y\right) \\
& \leq C(1-r)^{\varepsilon}\left(1+\int_{B(0, r)}(1-|y|)^{(\alpha-m-1) p+\varepsilon(p-1)-N+(N-1) / q} f_{3, x}(y)^{p(y)} d y\right)
\end{aligned}
$$

for $1 / 2<r<1$. Let $j_{0}$ be the smallest integer such that $r \leq 1-2^{-j_{0}-1}$. Note here that

$$
\begin{aligned}
& \int_{B(0, r)}(1-|y|)^{(\alpha-m-1) p+\varepsilon(p-1)-N+(N-1) / q} f_{3, x}(y)^{p(y)} d y \\
& \leq \sum_{j=0}^{j_{0}} \int_{B\left(0,1-2^{-j-1}\right) \backslash B\left(0,1-2^{-j}\right)}(1-|y|)^{(\alpha-m-1) p+\varepsilon(p-1)-N+(N-1) / q} f_{3, x}(y)^{p(y)} d y \\
& \leq C \sum_{j=0}^{j_{0}} 2^{-j((\alpha-m-1) p+\varepsilon(p-1)-N+(N-1) / q)} \int_{B\left(0,1-2^{-j-1}\right) \backslash B\left(0,1-2^{-j}\right)} f_{3, x}(y)^{p(y)} d y \\
& \leq C \sum_{j=0}^{j_{0}} 2^{-j((\alpha-m-1) p+\varepsilon(p-1)-N+(N-1) / q)} \omega\left(2^{-j}\right)^{-p} \\
& \leq C(1-r)^{(\alpha-m-1) p+\varepsilon(p-1)-N+(N-1) / q} \omega(1-r)^{-p}
\end{aligned}
$$

for $1 / 2<r<1$ by $(\omega 4)$, which gives the assertion.

We are now ready to show our main result.

Theorem 4.6. Let $1 \leq q<\infty$. Suppose $(\omega 4)$ holds for some $\varepsilon_{0}>0$.

(1) If

$$
N-\alpha p-1<(N-1) / q \leq N-\alpha p,
$$

then there exists a constant $C>0$ such that

$$
\liminf _{r \rightarrow 1-}(1-r)^{N-(\alpha-m-1) p-(N-1) / q} \omega(1-r)^{p} S_{q}\left(\left|K_{\alpha, m} f\right|^{p(r)}, r\right) \leq C
$$

for all nonnegative measurable functions $f$ with $\|f\|_{M^{p(\cdot), m+1, \omega}(\mathbf{B})} \leq 1$. 
(2) If

$$
N-\alpha p<(N-1) / q<N-(\alpha-m-1) p,
$$

then there exist constants $C>0$ and $1 / 2<r_{0}<1$ such that

$$
S_{q}\left(\left|K_{\alpha, m} f\right|^{p(r)}, r\right) \leq C(1-r)^{(\alpha-m-1) p-N+(N-1) / q} \omega(1-r)^{-p}
$$

for all $r_{0}<r<1$ and nonnegative measurable functions $f$ with $\|f\|_{M^{p(\cdot), m+1, \omega}(\mathbf{B})}$ $\leq 1$.

Proof. Let $f$ be a nonnegative measurable function with $\|f\|_{M^{p(\cdot), m+1, \omega}(\mathbf{B})} \leq 1$. For $x \in \mathbf{B}$, write

$$
K_{\alpha, m} f(x)=K_{1}(x)+K_{2}(x)+K_{3}(x)
$$

as before.

We first show assertion (1). Let $\varepsilon>0$ such that

$$
N-\alpha p-1+\varepsilon(2 p-1)<(N-1) / q<N-\alpha p+\varepsilon(2 p-1)<N-\alpha p+(m+1) p .
$$

Set

and

$$
d=-\alpha p+\varepsilon(2 p-1)+N-(N-1) / q
$$

$$
d(r)=-\alpha p(r)+\varepsilon(2 p(r)-1)+N-(N-1) / q .
$$

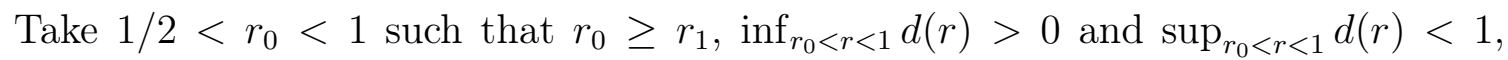
where $r_{1}$ is a constant appeared in Lemma 4.1. Let $r_{0}<r<1$ and $\beta>0$. First note by Lemma 4.3 that

$$
(1-r)^{N-(\alpha-m-1) p-(N-1) / q} \omega(1-r)^{p} S_{q}\left(\left|K_{2}\right|^{p(r)}, r\right) \leq C .
$$

By Lemma 4.4, we have

$$
(1-r)^{N-(\alpha-m-1) p-(N-1) / q} \omega(1-r)^{p} S_{q}\left(\left|K_{3}\right|^{p(r)}, r\right) \leq C .
$$

Finally, we obtain by Lemma 4.1 (1)

$$
\begin{aligned}
& S_{q}\left(\left|K_{1}\right|^{p(r)}, r\right) \\
& \leq C \omega(1-r)^{-p}\left\{(1-r)^{\beta}+\left.(1-r)^{\varepsilon(2 p-1)-(m+1) p} \int_{A(0, r)}|r-| y\right|^{-d(r)} g(y) d y\right\},
\end{aligned}
$$

where $g(y)=\left(\omega(1-|y|)(1-|y|)^{m+1} f(y)\right)^{p(y)}$.

Note here that

$$
\int_{A(r)} g(y) d y \leq C \omega(1-r)^{p} \int_{A(r)}\left((1-|y|)^{m+1} f(y)\right)^{p(y)} d y \leq C .
$$

Therefore

$$
\begin{aligned}
& (1-r)^{N-(\alpha-m-1) p-(N-1) / q} \omega(1-r)^{p} S_{q}\left(\left|K_{1}\right|^{p(r)}, r\right) \\
& \leq C\left\{(1-r)^{N-(\alpha-m-1) p-(N-1) / q+\beta}+\left.(1-r)^{d} \int_{A(0, r)}|r-| y\right|^{-d(r)} g(y) d y\right\} .
\end{aligned}
$$

In view of Lemma 4.2 , we can find a sequence $\left\{r_{j}\right\}$ of positive numbers and a positive integer $j_{0}$ such that $r_{j_{0}} \geq r_{0}, 1-2^{-j+1}<r_{j}<1-2^{-j}$ and

$$
\sup _{j \geq j_{0}}\left(1-r_{j}\right)^{N-(\alpha-m-1) p-(N-1) / q} \omega\left(1-r_{j}\right)^{p} S_{q}\left(\left|K_{1}\right|^{p\left(r_{j}\right)}, r_{j}\right) \leq C,
$$

which proves assertion (1).

Assertion (2) is obtained by Lemmas 4.1 (2), 4.3 and 4.4 . 
Setting $M^{p(\cdot), m+1, \omega}(\mathbf{B})=M^{p, m+1, \nu}(\mathbf{B})$ when $p(x)=p$ and $\omega(r)=r^{-\nu}$ with $\nu \geq 0$, we obtain the following corollary.

Corollary 4.7. Let $1 \leq p \leq q<\infty$.

(1) If

and

$$
\frac{1}{q}<\frac{N-(\alpha+\nu-m-1) p}{p(N-1)}
$$

$$
\frac{N-\alpha p-1}{p(N-1)}<\frac{1}{q} \leq \frac{N-\alpha p}{p(N-1)}
$$

then there exists a constant $C>0$ such that

$$
\liminf _{r \rightarrow 1-}(1-r)^{N / p-\alpha-\nu+m+1-(N-1) / q} S_{q}\left(\left|K_{\alpha, m} f\right|, r\right) \leq C
$$

(2) If

for all nonnegative measurable functions $f$ with $\|f\|_{M^{p, m+1, \nu}(\mathbf{B})} \leq 1$.

$$
\frac{N-\alpha p}{p(N-1)}<\frac{1}{q}<\frac{N-(\alpha+\nu-m-1) p}{p(N-1)}
$$

then there exists a constant $C>0$ such that

$$
\limsup _{r \rightarrow 1-}(1-r)^{N / p-\alpha-\nu+m+1-(N-1) / q} S_{q}\left(\left|K_{\alpha, m} f\right|, r\right) \leq C
$$

for all nonnegative measurable functions $f$ with $\|f\|_{M^{p, m+1, \nu}(\mathbf{B})} \leq 1$.

Remark 4.8. In Theorem 4.6 (1), "lim inf" can not be replaced by "lim sup". For this purpose, we first note from the proof of Lemma 2.2 (2) that

$$
K_{\alpha, m}(x, y) \geq C|x-y|^{\alpha-N}-C\left|x-y^{*}\right|^{\alpha-N} .
$$

Hence, if $0<\varepsilon<1$ is small enough, then

$$
K_{\alpha, m}(x, y) \geq 2^{-1}|x-y|^{\alpha-N} \quad \text { when }|x-y|<\varepsilon(1-|x|)
$$

since $1-|x| \leq\left|x-y^{*}\right|$.

Let $p>1$ and $1 \leq q<\infty$ satisfy

$$
\frac{1}{q}<\frac{N-\alpha p}{p(N-1)}
$$

and take $a \in \mathbf{R}$ such that

$$
\alpha+\frac{N-1}{q}<a \leq \frac{N}{p} .
$$

Let $r_{j}=2^{-j}$ for each positive integer $j$ and $\gamma>0$. Consider the function

$$
f(y)=\sum_{j=1}^{\infty}\left(j^{-\gamma} r_{j}\right)^{-a} \chi_{B_{j}},
$$

where $a<m+1+N / p, B_{j}=B\left(\left(1-r_{j}\right) \mathbf{e}, j^{-\gamma} r_{j+1}\right)$ and $\mathbf{e}=(1,0, \ldots, 0)$, and set

$$
u(x)=\int K_{\alpha, m}(x, y) f(y) d y .
$$

Then, for $0<r<1$, we have by $a \leq N / p$

$$
\begin{aligned}
\int_{C(0, r)}\left\{(1-|y|)^{m+1} f(y)\right\}^{p} d y & \leq C \sum_{j=j_{0}}^{\infty} r_{j}^{(m+1) p}\left(j^{-\gamma} r_{j}\right)^{-a p+N} \\
& \leq C(1-r)^{(m+1-a) p+N}\left(\log (e /(1-r))^{-\gamma(-a p+N)},\right.
\end{aligned}
$$


where $1-r<2^{-j_{0}} \leq 2(1-r)$, so that $f \in M^{p, m+1, \nu}(\mathbf{B})$ with $\nu=(m+1-a)+N / p>0$. For $x \in B_{j}$ with $|x|=1-r_{j}$, we have by Lemma $2.2(2)$ and $\alpha<a<N$

$$
\begin{aligned}
u(x) & \geq C \int_{B_{j}}|x-y|^{\alpha-N} f(y) d y-C \int_{\mathbf{B} \backslash B_{j}}|x-y|^{\alpha-N} f(y) d y \\
& \geq C\left(j^{-\gamma} r_{j}\right)^{-a+\alpha}-C \sum_{k \neq j}\left|r_{j}-r_{k}\right|^{\alpha-N}\left(k^{-\gamma} r_{k}\right)^{N-a} \\
& \geq C\left(j^{-\gamma} r_{j}\right)^{-a+\alpha}-C \sum_{k<j} r_{k}^{\alpha-N}\left(k^{-\gamma} r_{k}\right)^{N-a}-C \sum_{k>j} r_{j}^{\alpha-N}\left(k^{-\gamma} r_{k}\right)^{N-a} \\
& \geq C\left(j^{-\gamma} r_{j}\right)^{-a+\alpha}-C r_{j}^{\alpha-N}\left(j^{-\gamma} r_{j}\right)^{N-a}-C r_{j}^{\alpha-N}\left(j^{-\gamma} r_{j}\right)^{N-a} \geq C\left(j^{-\gamma} r_{j}\right)^{-a+\alpha}
\end{aligned}
$$

when $j$ is large enough, so that

$$
\begin{aligned}
S_{q}\left(u, 1-r_{j}\right) & \geq C\left(\frac{1}{\left|S\left(0,1-r_{j}\right)\right|} \int_{S\left(0,1-r_{j}\right) \cap B_{j}}\left(j^{-\gamma} r_{j}\right)^{(-a+\alpha) q} d S(x)\right)^{1 / q} \\
& \geq C\left(j^{-\gamma} r_{j}\right)^{-a+\alpha+(N-1) / q}
\end{aligned}
$$

for large $j$. This gives

$$
\begin{aligned}
r_{j}^{N / p-(\alpha+\nu-m-1)-(N-1) / q} S_{q}\left(u, 1-r_{j}\right) & =r_{j}^{-(\alpha-a)-(N-1) / q} S_{q}\left(u, 1-r_{j}\right) \\
& \geq C j^{\gamma(a-\alpha-(N-1) / q)}
\end{aligned}
$$

for large $j$. Hence if $\alpha+(N-1) / q<a \leq N / p$, then

$$
\limsup _{r \rightarrow 1}(1-r)^{N / p-(\alpha+\nu-m-1)-(N-1) / q} S_{q}(u, r)=\infty,
$$

as required.

\section{References}

[1] Futamura, T., K. Kitaura, and Y. Mizuta: Riesz decomposition for superbiharmonic functions in the unit ball. - Hokkaido Math. J. 4, 2009, 683-700.

[2] Futamura, T., and Y. Mizuta: Maximal functions for Lebesgue spaces with variable exponent approaching 1. - Hiroshima Math. J. 36, 2006, 23-28.

[3] Gardiner, S. J.: Growth properties of $p$ th means of potentials in the unit ball. - Proc. Amer. Math. Soc. 103, 1988, 861-869.

[4] Mizuta, Y.: Continuity properties of potentials and Beppo-Levi-Deny functions. - Hiroshima Math. J. 23, 1993, 79-153.

[5] Mizuta, Y.: Potential theory in Euclidean spaces. - Gakkōtosyo, Tokyo, 1996.

[6] Mizuta, Y., T. Ohno, and T. Shimomura: Growth properties for Riesz potentials of functions in weighted variable $L^{p(\cdot)}$ spaces. - Nonlinear Anal. 162, 2017, 49-75.

[7] Mizuta, Y., T. Ohno, and T. Shimomura: Growth properties of potentials in central Morrey-Orlicz spaces on the unit ball. - Ann. Acad. Sci. Fenn. Math. 43:1, 2018, 21-46.

[8] Mizuta, Y., and T. Shimomura: Boundary limits of spherical means for BLD and monotone BLD functions in the unit ball. - Ann. Acad. Sci. Fenn. Math. 24:1, 1999, 45-60.

[9] Shimomura, T.: Growth properties of hyperplane integrals of Sobolev functions in a half space. - Osaka J. Math. 38, 2001, 759-773.

[10] Stoll, M.: Boundary limits of subharmonic functions in the unit disc. - Proc. Amer. Math. Soc. $93,1985,567-568$. 